\title{
Tracers to Monitor the Response to Chemotherapy: In Vitro Screening of Four Radiopharmaceuticals
}

\author{
Lioe-Fee de Geus-Oei, ${ }^{1}$ Julliëtte van Eerd-Vismale, ${ }^{1}$ Carla Molthoff, ${ }^{2}$ Frans Corstens, ${ }^{1}$ \\ Wim Oyen, ${ }^{1}$ and Otto Boerman ${ }^{1}$ \\ ${ }^{1}$ Department of Nuclear Medicine, University Medical Center, Nijmegen, The Netherlands \\ ${ }^{2}$ PET Center/Nuclear Medicine, VU University Medical Center, Amsterdam, The Netherlands
}

\begin{abstract}
Objectives: It has been postulated that radiopharmaceuticals can be used to predict the therapeutic response to (chemo)therapy, which could lead to individualized treatment regimens. In this study, ${ }^{18} \mathrm{~F}$-deoxyglucose, ${ }^{99 m}$ Tc-tetrofosmin, ${ }^{125}$ I-deoxyuridineribose, and ${ }^{125}$ I-methyltyrosine were tested for this purpose. Methods: The uterine sarcoma cell line MES-SA (MDR-) and its multidrug resistant variant, MES-SA/Dx5 (MDR+), were used. The MDR+ cells express high levels of P-glycoprotein, which makes them relatively resistant to various chemotherapeutic agents. Cells were cultured in the presence of escalating concentrations of doxorubicin, and the cellular uptake of the radiopharmaceuticals was determined. Results: Decreasing ${ }^{18} \mathrm{~F}$-deoxyglucose uptake at escalating doxorubicin concentrations reflected the chemosensitivity of the cells: ${ }^{18} \mathrm{~F}$-deoxyglucose uptake in the MDR-cells was reduced to $40 \%$ of the baseline level in the presence of $1 \mu M$ of doxorubicin, compared to $74 \%$ in the MDR + cells. The ${ }^{125 I-}$ deoxyuridineribose uptake in MDR-cells was reduced to $2 \%$ of the baseline level when cultured at a concentration of $1 \mu M$ of doxorubicin, while this was $79 \%$ in the MDR + cells. The same trend was observed with ${ }^{125}$ I-methyltyrosine. The enhanced doxorubicin chemosensitivity of MDR + cells in the presence of verapamil, a modulator of $P$-glycoprotein, was reflected by the reduced uptake of ${ }^{18} \mathrm{~F}$-deoxyglucose, ${ }^{125}$ I-deoxyuridineribose, and ${ }^{125}$ I-methyltyrosine. Furthermore, baseline ${ }^{99 m}$ Tc-tetrofosmin uptake in $M D R+$ cells was more than six-fold lower than in MDR-cells. Conclusion: In the presence of doxorubicin, the uptake of ${ }^{18} \mathrm{~F}$-deoxyglucose, ${ }^{125} \mathrm{I}$-deoxyuridineribose and, to a lesser extent, ${ }^{125}$ I-methyltyrosine is more pronouncedly reduced in MDR-cells than in MDR + cells. The reversal of doxorubicin-resistance of MDR+ cells by verapamil was also reflected by the uptake of ${ }^{18} \mathrm{~F}$-deoxyglucose, ${ }^{125} \mathrm{I}$-deoxyuridineribose, and ${ }^{125}$ I-methyltyrosine. ${ }^{99 m}$ Tc-tetrofosmin uptake reflected P-glycoprotein expression without exposure to doxorubicin.
\end{abstract}

Key words: multidrug resistance, ${ }^{18}$ fluorodeoxyglucose, ${ }^{99 \mathrm{~m}} \mathrm{Tc}$-tetrofosmin, ${ }^{125} \mathrm{I}$-deoxyuridineribose, ${ }^{125}$ I-methyltyrosine

Address reprint requests to: Lioe-Fee de Geus-Oei; Department of Nuclear Medicine (internal postal code 565), University Medical Center Nijmegen; P.O. Box 9101, NL6500 HB Nijmegen, The Netherlands; Tel.: +31-243614048; Fax: +31-24-3618942

E-mail: L.degeus-oei@nucmed.umcn.nl

\section{INTRODUCTION}

One of the major problems in the treatment of cancer with chemotherapeutics is the development of multidrug resistance (MDR) by tumor 
cells. It is of great clinical importance to identify those patients that will respond to particular anticancer agents and, therefore, to avoid unnecessary toxicity, (effective) therapy delay, and expense in nonresponsive patients. Particularly in the case of newer biological treatments involving cytostatic (in contrast to cytolytic) drugs, an extended period of observation is often required before significant changes in the patient's clinical status, or in the radiographic evaluations, are observed. Therefore, there is a need for a relevant measure of treatment response that could identify treatment "success" or "failure" much earlier than changes in tumor size or volume identified with computed tomography (CT) or magnetic resonance (MR). An early assessment of treatment response is critical in designing appropriate and individualized treatment regimens. ${ }^{1}$

Various mechanisms are responsible for multidrug resistance, one of the most important being the overexpression of a plasma membrane phosphoglycoprotein, $\mathrm{P}$-glycoprotein (P-gp), which is encoded by the MDR1 gene. P-gp is the transporter protein responsible for actively pumping cytotoxic agents out of the cell. Besides P-gp, alternative intracellular mechanisms of resistance have been reported, including intracellular entrapment, compartmentalization or redistribution by the multidrug resistance-associated protein (MRP) and the lungresistant protein (LRP), enhanced drug detoxification by glutathion-S transferase, changes in levels or activity of nuclear targets, such as topoisomerase II, and alterations in the control of apoptosis. ${ }^{2} \mathrm{Be}-$ cause the overexpression of $\mathrm{P}$-gp is the most important mechanism responsible for MDR, we focused on P-gp in this study.

The distribution of P-gp in many organs suggests that the expression of P-gp is actually a physiological protection mechanism of the human body. P-gp can provide protection by extruding toxins out of the cells into urine, bile, and the intestinal lumen. It appears to protect critical organs, such as the brain and the testes, against toxic compounds. The MDR1 gene expressed in several normal human tissues, is associated with secretory or barrier functions, and in some bone marrow and blood cells. ${ }^{3}$ Overexpression of P-gp may result in increased excretion and decreased retention of various MDR substrates, and thus decreased cytotoxic efficacy of anticancer agents. ${ }^{4}$

Chemotherapeutic agents such as anthracyclines (daunorubicin and doxorubicin), vinca alkaloids (vincristine and vinblastine), epipodophyllotoxins (etoposide), and taxanes (paclitaxel) are all substrates for P-gp and are extruded out of the cell by P-gp. Besides chemotherapeutic drugs, several other drugs are substrates for P-gp, such as cardiovascular drugs (verapamil and digoxin), and immunosuppressives (cyclosporin A, PSC833). It is not yet clearly understood by what mechanism P-gp is able to recognize such diverse compounds, and, most likely, more than one drug binding site is involved. ${ }^{5,6}$

It is of great relevance to identify patients with MDR resulting from overexpression of P-gp. Detection of protein and RNA expression for the MDR1 pump can be performed in human tumor samples using a variety of techniques, such as immunohistochemistry, quantitative autoradiography, and reverse transcriptase polymerase chain reaction. ${ }^{7-10}$ However, detection of P-gp does not necessarily provide any information about the function of these pumps in the respective tissues. Furthermore, serial tumor biopsies are not generally performed. Radiotracers that can visualize the activity of cellular efflux pumps noninvasively are, therefore, of interest. In addition, various radiopharmaceuticals can potentially be used to predict the therapeutic response to chemotherapy. In our study, we studied 4 radiopharmaceuticals $\left({ }^{18} \mathrm{~F}\right.$-deoxyglucose (=FDG), ${ }^{99 \mathrm{~m}}$ Tc-tetrofosmin (=TF), ${ }^{125}$ I-methyltyrosine $(=\mathrm{IMT})$, and ${ }^{125}$ I-deoxyuridineribose $(=\mathrm{IUdR}))$ for their ability to monitor the response of cancer cells to doxorubicin treatment.

The experiments were performed in the presence and absence of the calcium channel blocker verapamil, a potent modulator of P-gp. To inhibit P-gp-mediated extrusion of chemotherapeutic agents, this modulator can be used beside other modulators, such as the immunosuppressive agent cyclosporin A and PSC833. ${ }^{11}$ Verapamil most likely acts by binding competitively to binding sites on the MDR transport proteins or by altering the affinities of the recognition domains in those proteins. ${ }^{12}$ Being informed about the effect of a modulator on both tumor tissue and normal tissues in each individual patient before starting chemotherapy could be clinically useful. This may help to select patients who will eventually benefit most from the addition of a modulator to the chemotherapeutic regimen. ${ }^{13}$

\section{MATERIALS AND METHODS}

\section{Cell Lines}

The cell line MES-SA 1976 (ATCC, CRL-1976) and its multidrug resistant variant, MES-SA/Dx5 
1977 (ATCC, CRL-1977) were used in these experiments. The development and characterization of the human uterine sarcoma cell line MES-SA and its multistep-selected MDR variant Dx 5 cells have been described elsewhere. ${ }^{14,15}$ In brief, wild-type MES-SA 1976 (P-gp-negative) cells were derived from a human uterine sarcoma. MES-SA/Dx5 1977 (P-gp-positive) cells were derived from MES-SA 1976 cells grown in the presence of increasing doxorubicin concentrations. As a result of doxorubicin exposure, MESSA/Dx5 1977 cells express high levels of MDR1 RNA and P-gp (but not MRP), and are relatively resistant to doxorubicin, as well as to various other chemotherapeutic agents. Doxorubicin resistance in this cell line is stable, despite long periods of growth in a drug-free medium.

\section{Radiopharmaceuticals}

${ }^{18}$ Fluoro-2-deoxy-D-glucose (FDG)

${ }^{18}$ Fluoro-2-deoxy-D-glucose was obtained commercially from Tyco Health B.V., Petten, The Netherlands.

\section{${ }^{99 m}$ Tc-Tetrofosmin (TF)}

Tetrofosmin kits were obtained commercially (Myoview $^{\circledR}$, Amersham Healthcare, Buckinghamshire, England UK). The labeling and quality control procedures were carried out according to the manufacturers instructions. The radiochemical purity of TF used in this study was consistently higher than $95 \%$.

\section{${ }^{125}$ I-Iodo-methyl-tyrosine (IMT)}

Synthesis of IMT was carried out essentially as described by Krummeich et al. ${ }^{16} 133 \mu \mathrm{L}$ of Iodogen $(0.75 \mathrm{mg} / \mathrm{mL})$ was added in a glass reaction vial (Iodogen tube), and the chloroform was evaporated under a stream of nitrogen. $400 \mu \mathrm{L} 0.11 \mathrm{M}$ borate buffer, $\mathrm{pH} 8.0$, and $90 \mu \mathrm{L}$ of L-alphamethyl tyrosine (Sigma-Aldrich Chemie b.v., Zwijndrecht, The Netherlands) dissolved in $0.22 \mathrm{M}$ borate buffer, $\mathrm{pH} 1.7$, were added. Subsequently, 200-250 $\mu \mathrm{Ci}$ of $\mathrm{Na}^{-125}{ }^{12}$ was added. The reaction was allowed to proceed for 10 minutes at room temperature. The reaction mixture was transferred into a $1.5-\mathrm{mL}$ vial and $250 \mu \mathrm{L}$ of $\mathrm{Na}_{2} \mathrm{~S}_{2} \mathrm{O}_{5}$ (2 $\mathrm{mg} / \mathrm{mL}$ ) in $0.11 \mathrm{M}$ borate buffer, $\mathrm{pH} 8.0$, was added. A Sep-Pak C-18 cartridge (Waters Corporation, Milford, MA) was activated with $15 \mathrm{~mL}$ of ethanol and rinsed with $15 \mathrm{~mL} \mathrm{5 \%}$ ethanol in $0.9 \%$ $\mathrm{NaCl}$. The reaction mixture was loaded onto the conditioned Sep-Pak cartridge. The Sep-Pak cartridge was eluted with $13 \mathrm{~mL} \mathrm{5 \%}$ ethanol in $0.9 \%$ $\mathrm{NaCl}$, and fractions of $1 \mathrm{~mL}$ were collected. The carrier-free IMT eluted in the fraction 4-6. The RCP of the IMT was analyzed using an HPLC system with C-18 column (Zorbax Rx-C18, $4.6 \times 25$ $\mathrm{cm}$ ) equipped with a radiodetector. $\mathrm{H}_{2} \mathrm{O}$ /ethanol/acetic acid $(92.5 / 5 / 2.5)$ was used as eluens with a flow of $0.5 \mathrm{~mL} / \mathrm{min}$. Rf values were: L- $\alpha$-methyl tyrosine precursor, 2.75 minutes (UV $280 \mathrm{~nm}$ ), free ${ }^{125} \mathrm{I} 3.1 \mathrm{~min}$ and ${ }^{125} \mathrm{I}$-IMT product, $10.1 \mathrm{~min}$. Radiochemical yield was higher than $95 \%$, with a specific activity of $3612 \mathrm{GBq} / \mathrm{mol}$.

\section{5-[ ${ }^{125}$ I]Iodo-2'-deoxyuridine (IUdR)}

Noncarrier-added sterile 5-[ ${ }^{125}$ I]Iodo-2'-deoxyuridine (IUdR) (specific activity $74 \mathrm{TBq} / \mathrm{mmol}$ ) was purchased from Amersham International (Buckinghamshire, England UK).

\section{Drugs}

Doxorubicin hydrochloride was obtained as Adriblastina RTU ( $2 \mathrm{mg} / \mathrm{mL}$ for injection) (Pharmacia and Upjohn, Woerden, The Netherlands). It was stored at $4{ }^{\circ} \mathrm{C}$ and protected from light. Verapamil hydrochloride $(2.5 \mathrm{mg} / \mathrm{mL})$ was purchased from Knoll AG, BASF Pharma, Ludwigshafen, Germany.

\section{In Vitro Assay}

Cells were cultured at $37^{\circ} \mathrm{C}, 5 \% \mathrm{CO}_{2}$, in RPMI1640 (Gibco, Life Technologies, Gaithersburg, MD) with $10 \%$ fetal calf serum (FCS) and $2 \mathrm{mM}$ glutamine, using $162 \mathrm{~cm}^{2}$ culture flasks. Cells $\left(10^{5}\right.$ cells $\left./ \mathrm{mL}\right)$ were seeded in 24 -well plates. The aim of the first series of experiments was to determine the optimal doxorubicin incubation schedule. Doxorubicin (escalating concentrations $0-1000 \mu \mathrm{M}$ ) was added starting at 72,48 , and 24 hours before the addition of the radiopharmaceuticals, as presented in Figure 1. Subsequently, the medium was removed from the wells, and cells were washed twice with saline. To each well, $10^{6} \mathrm{cpm} / \mathrm{mL} \quad(1 \mathrm{~mL} / \mathrm{well}){ }^{125} \mathrm{I}$-deoxyuridineribose in RPMI-1640 $+0.5 \%$ BSA (Gibco, Invitrogen, Breda, The Netherlands), ${ }^{125}$ I-methyltyrosine in RPMI-1640 + 0.5\% BSA, ${ }^{18} \mathrm{~F}$-deoxyglucose in glucose-free-DMEM + $0.5 \%$ BSA or ${ }^{99 m}$ Tc-tetrofosmin in RPMI$1640+0.5 \%$ BSA, respectively, was added. After 1 hour of incubation at $37^{\circ} \mathrm{C}$, cells were washed, swabbed, and counted in a well-type gamma counter. The mean cell-associated activ- 


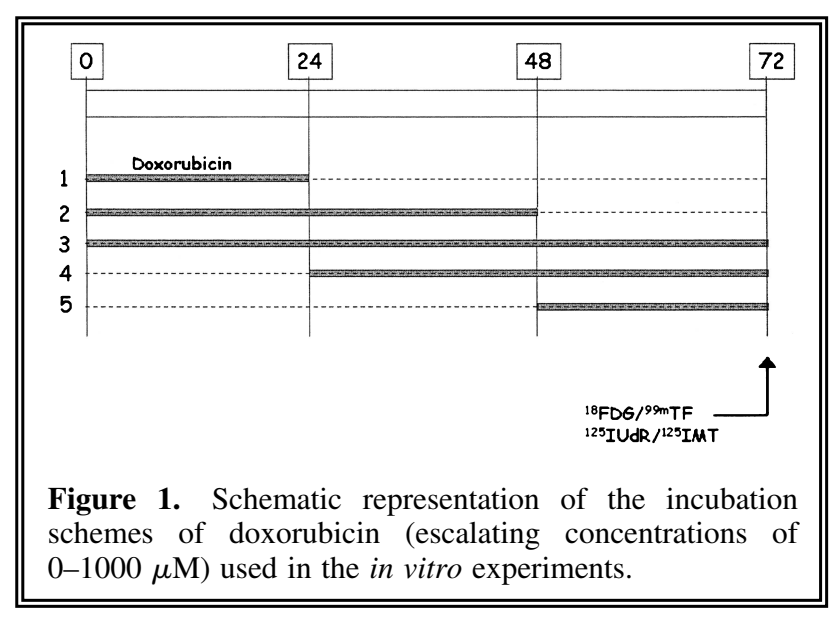

ity of each of the radiopharmaceuticals following incubation in the absence of doxorubicin was set at $100 \%$. The uptake at the various doxorubicin concentrations was expressed as the percentage of this activity.

\section{MTT Assay}

In order to investigate whether tracer uptake indeed reflected cell proliferation, the mitochondrial dehydrogenase activity and, hence, cell survival, was measured using the MTT assay (3-[4.5-dimethylthiazol-2-yl]-2.5-diphenyltetrazolium bromide, Thiazolyl blue [Sigma Chemical Co., St. Louis, MO). Dissolved MTT is converted to an insoluble purple formazan by cleavage of the tetrazolium ring by mitochondrial dehydrogenases of viable cells, and the absorbance of the solubilized formazan solution is proportional to the number of viable cells. Cell surivival of both cell lines (MES-SA 1976 and MES-SA/Dx5 1977) at doxorubicin concentrations of $0-1 \mu \mathrm{M}$, was expressed relative to their controls in the absence of any treatment. Cells were seeded into 24-well plates (in triplicate) at a density of approximately $3 \times 10^{4}$ cells per well in RPMI-1640, with $10 \%$ FCS and $2 \mathrm{mM}$ glutamine for 48 hours. At 48 hours, the medium was replaced by doxorubicin (concentrations 0-1 $\mu \mathrm{M})$ in RPMI-1640 for an exposure period of 48 hours. Then, cells were washed with saline, and the MTT stock solution $(5 \mathrm{mg} / \mathrm{mL}$ in RPMI-1640 without phenol red with $10 \%$ FCS and $2 \mathrm{mM}$ glutamine) was added to each well $(320 \mu \mathrm{L} /$ well $)$ and incubated for 5 hours at $37^{\circ} \mathrm{C}$. At the end of the incubation period, the medium was removed, cells were washed twice with saline, and the converted dye was solubilized with $1 \mathrm{~mL} 0.01 \mathrm{~N}$
$\mathrm{HCl} / 10 \%$ SDS during 16 hours at $37{ }^{\circ} \mathrm{C}$. Absorbance of converted dye was measured at a wavelength of $570 \mathrm{~nm}$.

\section{RESULTS}

\section{MTT Assay}

MTT results provide evidence that 48 hours of exposure to doxorubicin could induce reproducible cell death at escalating doxorubicin concentrations $(0-1 \mathrm{mM})$ to an increasing extent, with an obvious difference between the MDRcells and MDR + cells. Measurements of relative cell survival compared to untreated control cells showed that doxorubicin induced $80 \%$ cell death at $1 \mu \mathrm{M}$ in the MDR - cells, compared to only $5 \%$ at $1 \mu \mathrm{M}$ in the MDR + cells.

\section{Experiments to Determine the Most Optimal Incubation Scheme}

The aim of the first series of experiments was to determine the schedule to expose cancer cells to doxorubicin that would lead to the most pronounced effect on the uptake of each of the radiopharmaceuticals. Figure 2 demonstrates the results of the 5 different doxorubicin incubation schemes measured with FDG. The most pronounced difference in reduction of FDG uptake between the MDR + and MDR - cell line at low doxorubicin dose levels $(0.1-1.0(\mu \mathrm{M})$ was seen with the incubation schemes 2,3 , and 4 . This also applied for IMT and IUdR. Based on these observations, scheme number 4 (48 hours doxorubicin exposure, followed by 1 hour incubation with each of the radiopharmaceuticals) was chosen for future experiments. In this scheme, there is no delay between incubation with doxorubicin and addition of radiopharmaceuticals, avoiding tumor-cell recovery.

The FDG uptake at escalating doxorubicin doses (Fig. 2) correlated with the chemosensitivity of the cells: FDG uptake of the MDR - cells declined at lower doxorubicin concentrations, compared to that of MDR + cells. To reach the same reduction of FDG uptake, a 10-fold higher doxorubicin concentration was needed in the MDR + cells. Higher doxorubicin levels $(>100$ $(\mu \mathrm{M})$ were cytotoxic to both cell lines, regardless of their chemosensitivity.

The results from the IUdR and IMT experiments showed an even more pronounced effect (Fig. 3). In the chemosensitive cells, IUdR uptake was reduced to zero at a 100 -fold lower dose 

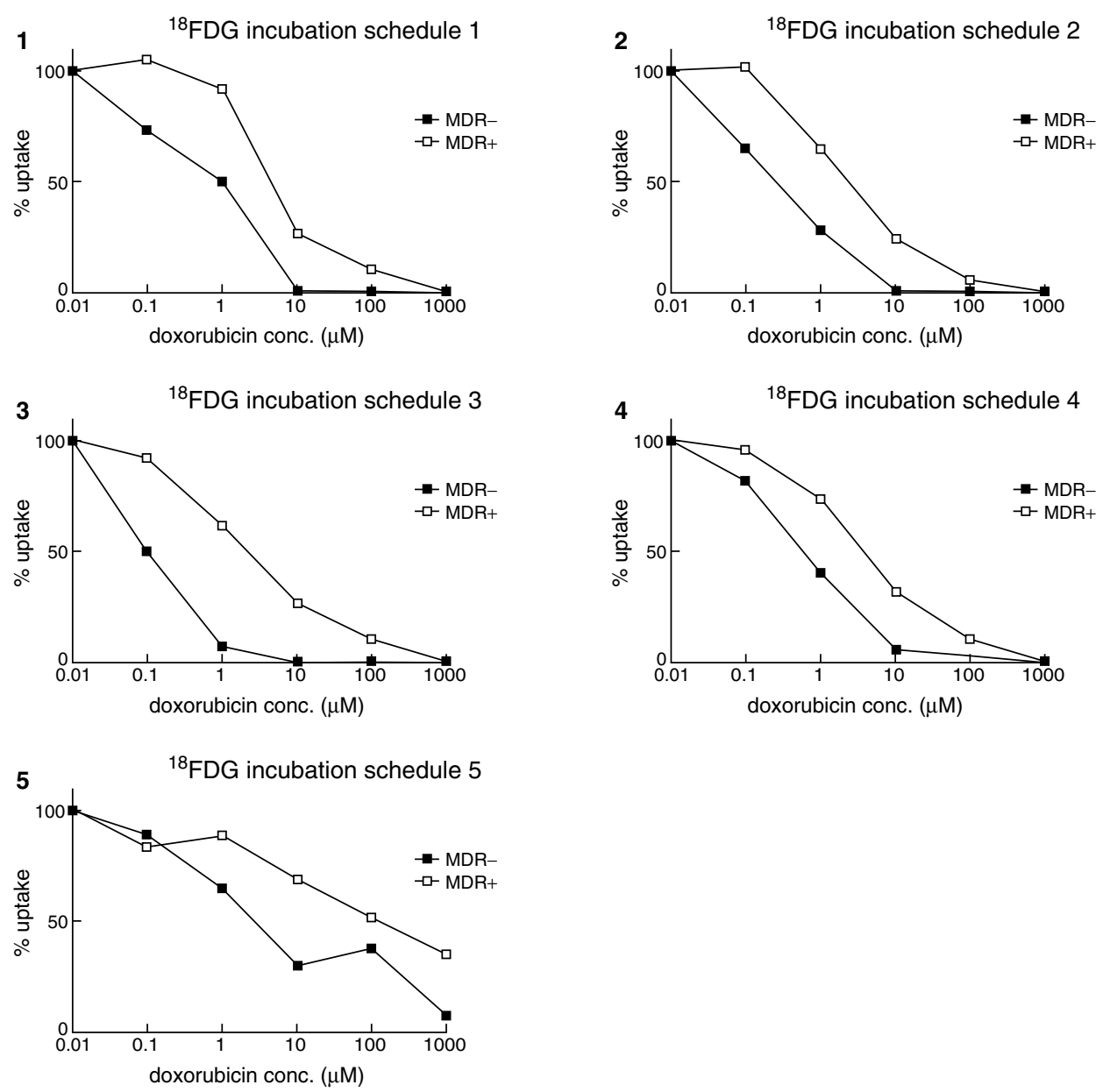

Figure 2. These graphs demonstrate the results of the 5 different doxorubicin incubation schemes, as shown in Figure 1, measured with FDG.

than the chemoresistant cells ( $1 \mu \mathrm{M}$ compared to $100 \mu \mathrm{M})$. With IMT, this was almost a 1,000fold $(1 \mu \mathrm{M}$, compared to $1,000 \mu \mathrm{M})$.

99m Tc-Tetrofosmin (TF) differed from the other three radiotracers in some aspects. Because the amount of uptake of this tracer reflects the Pgp activity, there is no need to expose the cells to doxorubicin in the assay. In order to measure P-gp activity, just the percentage of uptake of TF in the absence of doxorubicin are of interest. Baseline TF uptake of the MDR + cells was more than sixfold lower compared to that in MDRcells, presumably resulting from enhanced P-gp activity of MDR + cells.

\section{Effects of Verapamil}

In the second series of experiments, the effect of the P-gp antagonist verapamil on the effect of dox- orubicin on tracer uptake was investigated. In the first experiment, a suitable verapamil concentration was determined for these experiments. Both cell lines were incubated with escalating verapamil concentrations (1-125 $\mu \mathrm{g} / \mathrm{mL}$ ) in the absence of doxorubicin (Fig. 4). At the two highest verapamil concentrations tested ( 25 and $125 \mu \mathrm{g} / \mathrm{mL}$ ), the FDG uptake of the cells was reduced and, therefore, 5 $\mu \mathrm{g} / \mathrm{mL}$ verapamil was used in further experiments.

Subsequently, the effect of verapamil (5 $\mu \mathrm{g} / \mathrm{mL}$ ) on cell viability at various doxorubicin concentrations was tested in the MTT assay (Fig. 5). Verapamil had only a limited effect on the number of viable cells. In the presence of verapamil, the MDR + cells were more susceptible to high concentrations of doxorubicin (10-100 $\mu \mathrm{M})$. Interestingly, the number of MDR - cells at low doxorubicin concentrations $(0.1-1 \mu \mathrm{M})$ was also reduced by verapamil. Verapamil only 

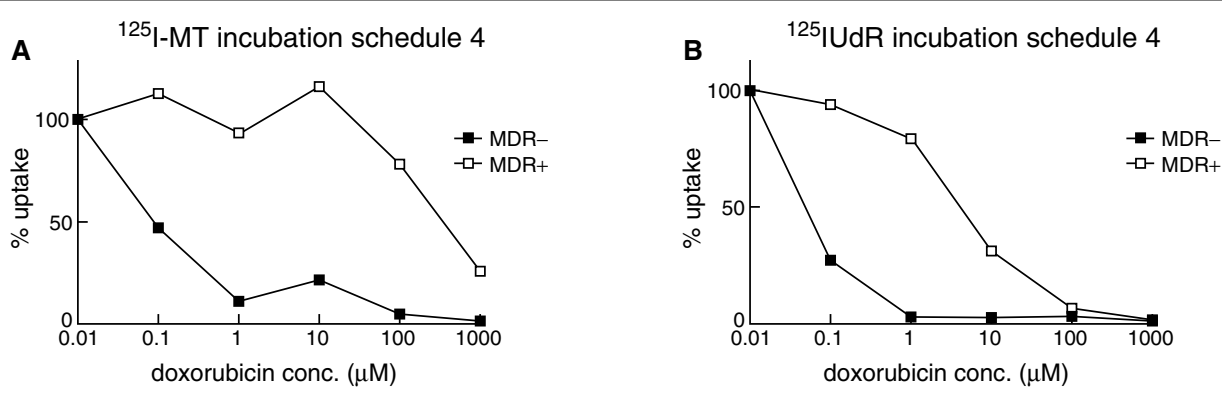

Figure 3. Results of doxorubicin incubation scheme 4 (see Figure 1). Measurements with ${ }^{125}$ I-methyltyrosine (A) and ${ }^{125}$ IUdR (B).

slightly affected cell viability when cultured in the presence of doxorubicin.

In contrast, the reversal of the drug-resistance by verapamil was clearly reflected by the uptake of FDG, IUdR, and IMT. Fig 6A shows that FDG uptake in the MDR + cells was markedly reduced because of the presence of verapamil. In fact, based on the FDG uptake the MDR + cells became almost as sensitive to doxorubicin as the MDR - cells. The uptake of FDG was markedly reduced in the MDR + cells when verapamil was coincubated: from $79 \%$ to $18 \%$ at $1.0 \mu \mathrm{M}$ doxorubicin. Although less pronounced, this effect of verapamil was also observed with the MDRcells: from $48 \%$ to $3 \%$ at $1.0 \mu \mathrm{M}$ doxorubicin. The same trend was observed with IUdR and IMT (Fig. 6B and 6C). The effects on TF uptake were less apparant (Fig. 7). In the absence of verapamil, the uptake of TF was $0.13 \%$ ID in the MDR + cells, compared to $0.87 \%$ ID in the

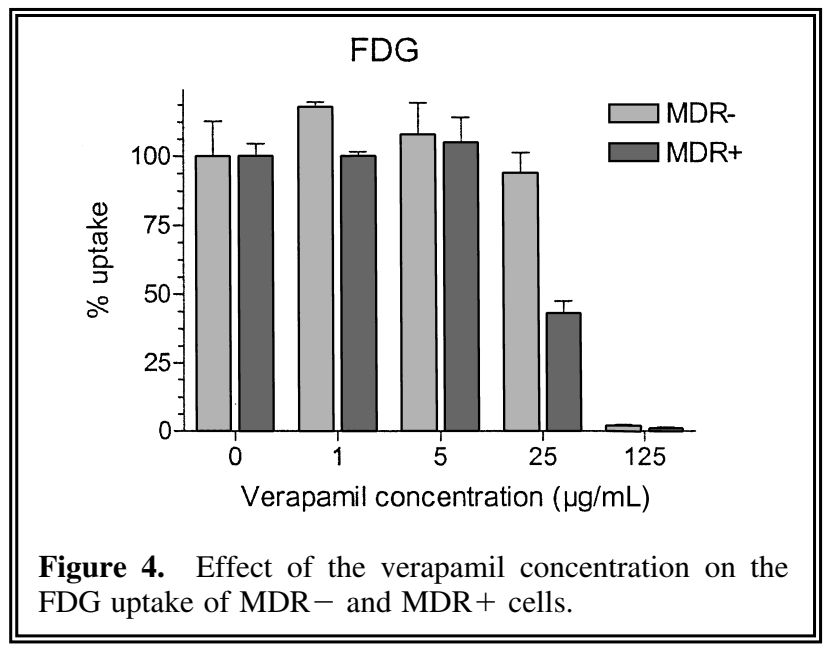

MDR - cells, whereas in the presence of verapamil these values were $0.19 \%$ ID and $1.43 \%$ ID, respectively.

\section{DISCUSSION}

The results of this study show that ${ }^{18}$ Fluoro-2deoxy-D-glucose (=FDG), 5-[ $\left.{ }^{125} \mathrm{I}\right]$ Iodo-2'-deoxyuridine $(=\mathrm{IUdR})$, and ${ }^{125}$ I-Iodo-methyl-tyrosine (=IMT) uptake is more pronouncedly reduced after exposure to doxorubicin in MES-SA cells that are drug-sensitive, as compared to MES-SA cells that are drug-resistant. The difference between the sensitive and resistant cell line was displayed most pronouncedly by IUdR. The results from the MTT assay showed that tracer uptake reflected cell viability. Exposure to doxorubicin induced a reduction in cell mitochondrial dehydrogenase activity, which was more pronounced in the MDR - cells. In these assays, the radiopharmaceuticals FDG, IMT, and IUdR had similar features. First, all showed a clear discrimination in uptake pattern between the sensitive and the resistant cell line. Second, the effect was reversed by the addition of a P-gp-modulator. The three radiopharmaceuticals also had various distinctive features, which makes each of them unique.

Radiopharmaceuticals originally developed for diagnosis of cardiac viability could be used as a sensitive tool for the detection of MDR. ${ }^{99 \mathrm{~m}}$ Tc-tetrofosmin (= TF) was the first radiotracer studied for this application. As compared to FDG, IMT, and IUdR, TF potentially has the advantage that it can be used before starting chemotherapy. The other three radiopharmaceuti- 


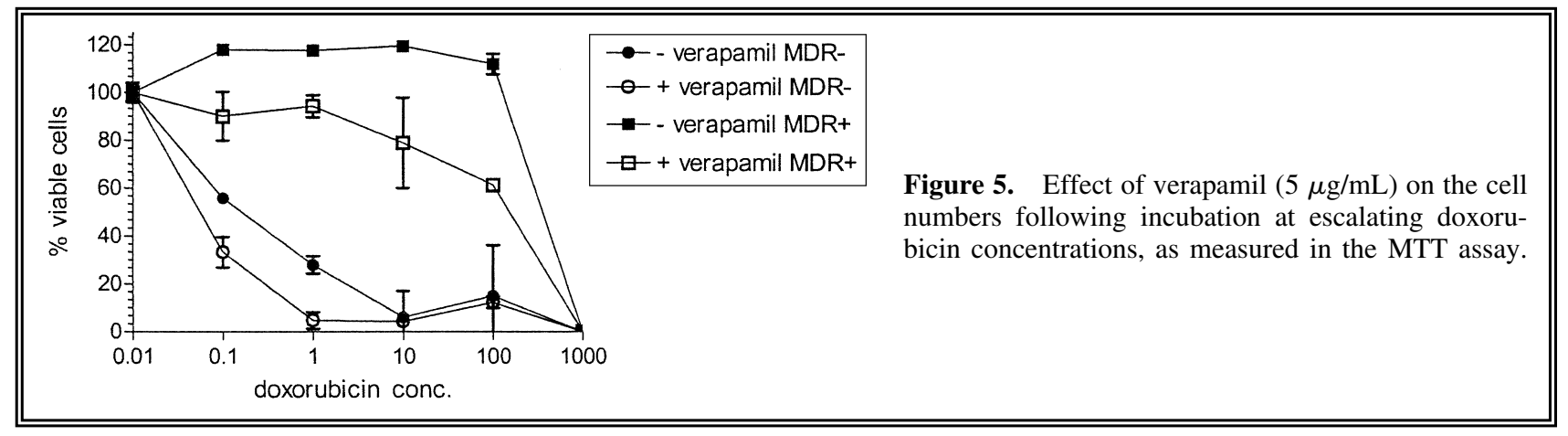

cals reflect the change in cell metabolism or proliferating activity after exposure to doxorubicin, which is essentially different from TF-accumulation, which is based on functional imaging of P-gp. Our results show that a reduced intracellular accumulation of TF is seen in resistant cells, as compared to their drug-sensitive counterpart. Unexpectedly, the reduced uptake of TF in MDR + cells was only minimally enhanced when verapamil was coincubated. Possibly because of the relatively high P-gp expression on these cells, verapamil could not effectively enhance intracellular TF accumulation. Interestingly, the MTT assay showed that coincubation of verapamil hardly affected the number of viable cells. In contrast, the cellular uptake of FDG, IMT, and IUdR was markedly reduced when verapamil was added, indicating that verapamil reduced the metabolic functions of the cells (glycolytic acitivity, aminoacid transport, and DNA synthesis, respecitively) rather than affecting the number of cells.

${ }^{18}$ Fluoro-2-deoxy-D-glucose (=FDG) was chosen as an indicator for cell metabolic activity. FDG enters normal and malignant cells using the same transport mechanisms as glucose and is trapped intracellularly because of its low dephosphorylation rate. The increased rate of glycolysis in tumor cells enhances the uptake of FDG relative to normal cells. The therapeutic effectiveness of cancer treatment by radiotherapy and chemotherapy is usually evaluated by morphologic changes in tumor size examined by $\mathrm{X}$ ray studies, CT, ultrasound, or MRI. An advantage of FDG-PET over these anatomic-structure imaging modalities, is the ability to distinguish active or recurrent disease and residual scar tissue after therapy. The most important advantage of FDG is that metabolic alterations of tumor cells, indicative of tumor response to therapy, may occur before alterations in tumor size. Our results show that FDG is able to differentiate between the MDR + cells and MDR - cells, i.e.,

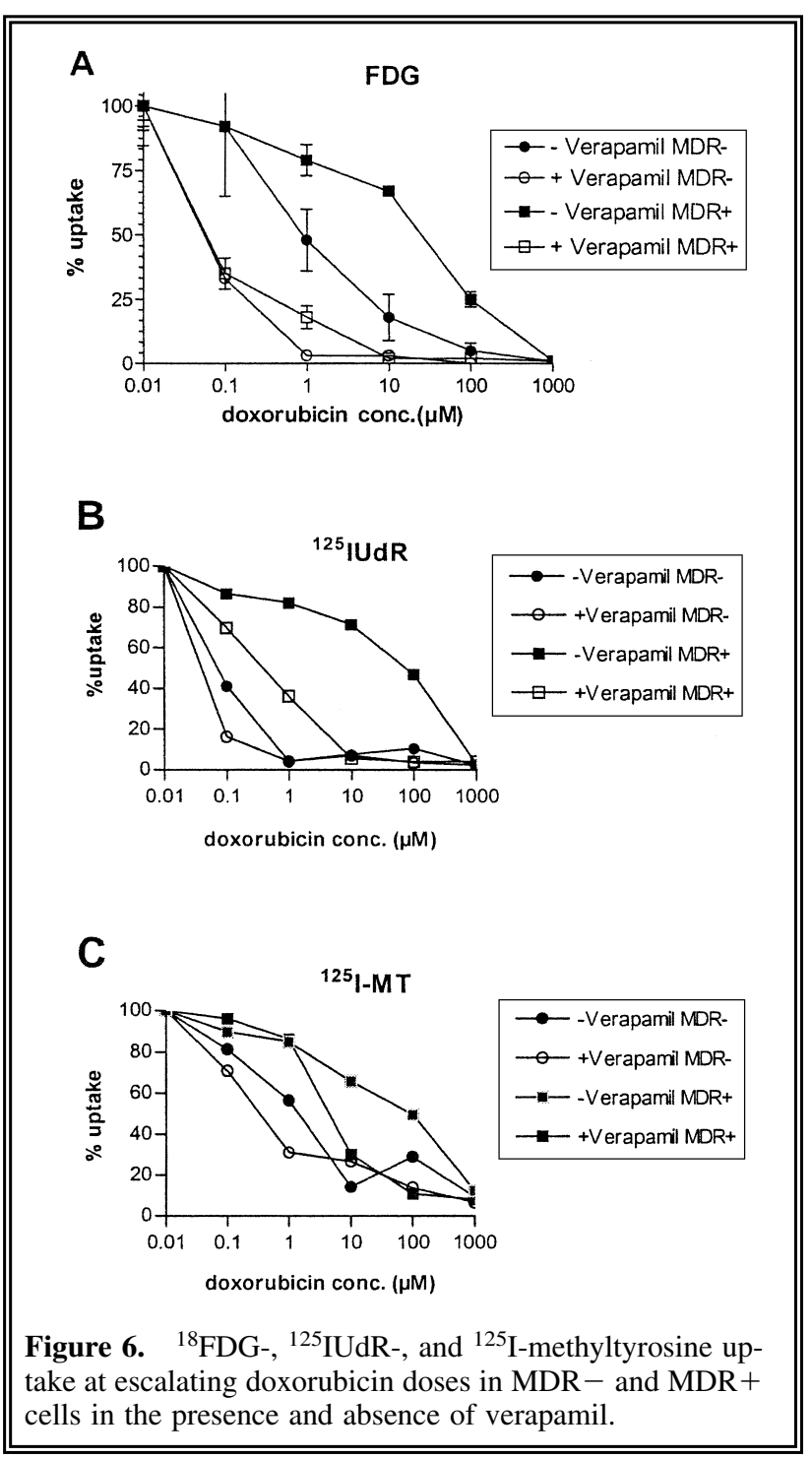




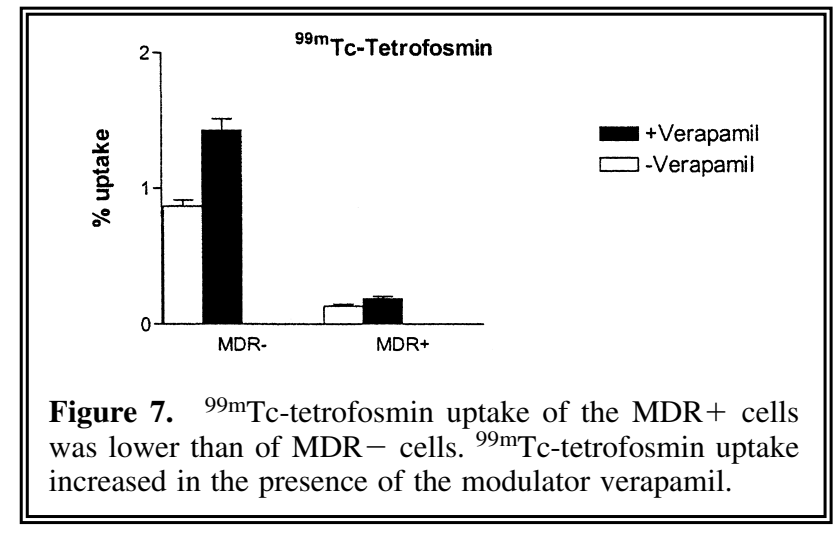

FDG uptake decreased more pronouncedly in the responding cells. Studies on small numbers of patients suggest that FDG uptake decreases in responding lesions, although a correlation between FDG uptake and the P-gp status of tumors has not yet been established. ${ }^{17-21}$

Another relevant target for functional tumor imaging is the uptake of amino acid analogs in cancer cells for which radiolabeled amino acids, such as ${ }^{125}$ I-Iodo-methyl-tyrosine (=IMT), can be applied. It is known that IMT shows a favorable normal distribution. Physiological low uptake is found in the brain, liver, and spleen, but intense uptake in the kidneys and urinary system, as it is renally excreted. ${ }^{22}$ The results of our in vitro experiments showed that the suppression of cell proliferation by doxorubicin can be measured using IMT.

The potential for obtaining functional images of DNA synthesis using PET and SPECT has been recognized for some time. ${ }^{23}$ Halogenated thymidine analogs such as IUdR can be used as cell proliferation markers for in vitro studies because these compounds are rapidly incorporated into newly synthesized DNA. In vivo, the use of IUdR is limited as a result of the moderate image quality and the inaccurate calculation of proliferation rates, because of its rapid in vivo degradation. The $\mathrm{C}-\mathrm{N}$ glycosidic bond of IUdR is not stable in vivo, which leads to metabolites with reduced tumor affinity. ${ }^{24}$ The attractive aspect of IUdR is that the uptake of IUdR reflects DNA synthesis and, hence, the proliferative status of tumors and normal tissues. In our experiments, IUdR displayed the most marked relative reduction in MES-SA cells that respond to exposure to doxorubicin, as compared to FDG and IMT.

Clinically, it is also important to detect and evaluate multidrug resistance transporter activity in tumors, as this could also be utilized as a tool in identifying patients who may benefit from combined therapy with P-gp or multidrug resistance-associated protein (MRP) modulators, as well as in the evaluation of the effectiveness of such treatment in vivo. It might also allow for the selection of the proper modulator for the individual patient. A number of drugs have been identified (e.g., calcium channel blockers, antiarrhythmics, antidepressants, and many others) that can reverse P-gp-mediated MDR. These drugs (e.g., verapamil, quinidine, and cyclosporin A) sensitize MDR tumor cells to coadministered cytotoxic agents. However, many of them are of limited clinical use because of side effects in the relevant doses. ${ }^{25}$ Clinical trials of inhibitors have often been hampered by the inability to document multidrug resistance in solid tumors of individual patients. ${ }^{26}$ It may be valuable to assess the effect of a modulator on both tumor tissue and normal tissues in each individual patient prior to treatment, not only to choose the most effective modulator or combination of modulators in clinical trails, but also to determine the most effective dose combined with the least toxicity of anticancer drugs. Response to doxorubicin was affected by the presence, or absence, of verapamil. This could clearly be measured with FDG and IMT, as well as with IUdR. However, in contrast to other studies, ${ }^{27}$ in our study, verapamil did not dramatically increase the accumulation of $\mathrm{TF}$ in the MDR + cells.

\section{CONCLUSION}

This study shows that FDG, IUdR, and IMT uptake is more pronouncedly reduced in MES-SA cells that respond to exposure to doxorubicin than in cells that do not. IUdR displayed the most markedly relative reduction. Unlike these three radiopharmaceuticals, TF shows these differences before exposure to doxorubicin, reflecting the expression of the MDR1 gene. The P-gp modulator, verapamil, is able to reverse the doxorubicin resistance, which could be measured with 3 out of 4 radiopharmaceuticals. These findings warrant comparative studies in in vivo tumor models.

\section{REFERENCES}

1. Blasberg RG, Roelcke U, Weinreich R, et al. Imaging brain tumor proliferative activity with $\left[{ }^{124} I\right]$ iododeoxyuridine. Cancer Res 2000;60:624.

2. Del Vecchio S, Ciarmiello A, Salvatore M. Clinical 
imaging of multidrug resistance in cancer. $Q \mathrm{~J} \mathrm{Nucl} \mathrm{Med}$ 1999;43:125.

3. Roninson IB. The role of the MDR1 (P-glycoprotein) gene in multidrug resistance in vitro and in vivo. Biochem Pharmacol 1992;43:95.

4. Levchenko A, Mehta BM, Lee JB, et al. Evaluation of $11 \mathrm{C}$-colchicine for PET imaging of multiple drug resistance. J Nucl Med 2000;41:493.

5. Tamai I, Safa AR. Azidopine noncompetitively interacts with vinblastine and cyclosporin A binding to $\mathrm{P}$ glycoprotein in multidrug resistant cells. $J$ Biol Chem 1991;266:16796.

6. Litman T, Zeuthen T, Skovsgaard T, et al. Competitive, noncompetitive, and cooperative interactions between substrates of P-glycoprotein as measured by its ATPase activity. Biochim Biophys Acta 1997;1361:169.

7. Nooter K, Stoter G. Molecular mechanisms of multidrug resistance in cancer chemotherapy. Pathol Res Pract 1996;192:768.

8. Beck WT, Grogan TM, Willman CL, et al. Methods to detect P-glycoprotein-associated multidrug resistance in patients' tumors: Consensus recommendations. Cancer Res 1996;56:3010.

9. Noonan KE, Beck C, Holzmayer TA, et al. Quantitative analysis of MDR1 (multidrug resistance) gene expression in human tumors by polymerase chain reaction. Proc Natl Acad Sci USA 1990;87:7160.

10. Herzog CE, Trepel JB, Mickley LA, et al. Various methods of analysis of mdr-1/P-glycoprotein in human colon cancer cell lines. J Natl Cancer Inst 1992;84:711.

11. Hendrikse NH, Franssen EJ, van der Graaf WT, et al. Visualization of multidrug resistance in vivo. Eur J Nucl Med 1999;26:283.

12. Sarkadi B, Muller M. Search for specific inhibitors of multidrug resistance in cancer. Semin Cancer Biol 1997;8:171.

13. Hendrikse NH, Franssen EJ, van der Graaf WT, et al. ${ }^{99 \mathrm{~m}} \mathrm{Tc}$-sestamibi is a substrate for P-glycoprotein and the multidrug resistance-associated protein. $\mathrm{Br} \mathrm{J} \mathrm{Can-}$ cer 1998;77:353.

14. Harker WG, Sikic BI. Multidrug (pleiotropic) resistance in doxorubicin-selected variants of the human sarcoma cell line MES-SA. Cancer Res 1985;45:4091.

15. Harker WG, MacKintosh FR, Sikic BI. Development and characterization of a human sarcoma cell line, MES-SA, sensitive to multiple drugs. Cancer Res 1983;43:4943.
16. Krummeich C, Holschbach M, Stocklin G. Direct n.c.a. electrophilic radioiodination of tyrosine analogues: Their in vivo stability and brain-uptake in mice. Appl Radiat Isot 1994;45:929.

17. Bender H, Bangard N, Metten N, et al. Possible role of FDG-PET in the early prediction of therapy outcome in liver metastases of colorectal cancer. Hybridoma 1999;18:87.

18. Haberkorn U, Strauss LG, Dimitrakopoulou A, et al. Fluorodeoxyglucose imaging of advanced head and neck cancer after chemotherapy. $J$ Nucl Med 1993;34:12.

19. Schelling M, Avril N, Nahrig J, et al. Positron emission tomography using [(18)F]fluorodeoxyglucose for monitoring primary chemotherapy in breast cancer. J Clin Oncol 2000;18:1689.

20. Smith IC, Welch AE, Hutcheon AW, et al. Positron emission tomography using [(18)F]-fluorodeoxy-D-glucose to predict the pathologic response of breast cancer to primary chemotherapy. J Clin Oncol 2000;18:1676.

21. Bassa P, Kim EE, Inoue T, et al. Evaluation of preoperative chemotherapy using PET with fluorine-18-fluorode-oxyglucose in breast cancer. $\mathrm{J} \mathrm{Nucl} \mathrm{Med}$ 1996;37:931.

22. Jager PL, Vaalburg W, Pruim J, et al. Radiolabeled amino acids: Basic aspects and clinical applications in oncology. J Nucl Med 2001;42:432.

23. Christman D, Crawford EJ, Friedkin M, et al. Detection of DNA synthesis in intact organisms with positronemitting (methyl-11 C)thymidine. Proc Natl Acad Sci USA 1972;69:988.

24. Toyohara J, Hayashi A, Sato M, et al. Rationale of 5-(125)i-iodo-4'-thio-2'-deoxyuridine as a potential iodinated proliferation marker. J Nucl Med 2002;43:1218.

25. Scheulen ME. Clinical relevance of P-glycoprotein with respect to the application of resistance modifiers. Int $J$ Clin Pharmacol Ther 1998;36:41.

26. Utsunomiya K, Ballinger JR, Piquette-Miller M, et al. Comparison of the accumulation and efflux kinetics of technetium-99m sestamibi and technetium-99m tetrofosmin in an MRP-expressing tumor cell line. Eur $J$ Nucl Med 2000;27:1786.

27. Moretti JL, Caglar M, Duran-Cordobes M, et al. Can nuclear medicine predict response to chemotherapy? Eur J Nucl Med 1995;22:97. 